\title{
Aspects of Weight-Support Mechanisms in Rehabilitation Robotics.
}

\author{
Arno H.A. Stienen (PhD) and Edsko E.G. Hekman (MSc) and Herman van der Kooij (PhD) \\ and Michael D. Ellis (PT, DPT) and Jules P.A. Dewald (PT, PhD)
}

\begin{abstract}
Weight support can facilitate upper-limb movements, with which the patients may do more and more meaningful exercises earlier in the rehabilitation process. Most rehabilitation devices support the arm against gravity in one way or the other. Weight support can be realized by limiting vertical displacement or applying constant supportive forces which counteract the gravitational pull. Of these, using constant supportive forces is the most natural way to facilitate natural arm movements as it allows full freedom of movement and the amount of weight support is scalable to the patients needs. To apply the supporting forces to the arm, endpoint mechanisms and exoskeletons are more complex to build and use then cable suspensions, but offer more control over the movements. Finally, passive weight support is inherently safe, but active systems have enhanced control options and the capability to create training conditions beyond limb weight.
\end{abstract}

\section{INTRODUCTION}

Supporting the arm against gravity of hemiparetic stroke patients has been shown to result in significant improvements in the arm function of patients [1], [2], [3], [4], [5]. In these training studies, the weight support facilitated functional movement of the patients and was gradually reduced as performance improved. Cross-sectional studies have shown that weight support does not negatively influence the execution of movements and only facilitates them [6], [7]. In these studies, weight support has been provided by exoskeletons [1], [3], endpoint manipulators [8], [2] and cable suspensions [9], [6], [7].

Of these devices, endpoint manipulators have a single connection to the hand, wrist or forearm. Often patients hold onto a handle while making movements in a virtual environment. Exoskeletons are external skeletons placed parallel to the arm and are generally powered by actuators on each of the joints. They control (a subset of) the joints of the shoulder, elbow, and wrist directly, at the cost of more complex mechanics. Cable suspensions support the arm with one or two slings, increasing both control options and complexity with every additional cable linkage. They are simplest to realize but offer the least amount of control of the movements of the arm.

These device types do differ in the resulting implementation of weight support, with each having their own ad-

This work was supported by National Institutes of Health (2R01HD039343-06A1: J. Dewald) and SenterNovem (NL, TSGE2050).

Stienen, Ellis and Dewald are with the Department of Physical Therapy \& Human Movement Sciences, Northwestern University, Chicago (IL), USA arnostienen@gmail.com.

Dewald is with the Departments of Physical Medicine \& Rehabilitation and Biomedical Engineering, Northwestern University, Chicago (IL), USA.

Stienen, Hekman, and Van der Kooij are with the Laboratory of Biomechanical Engineering, University of Twente, Enschede, NL.

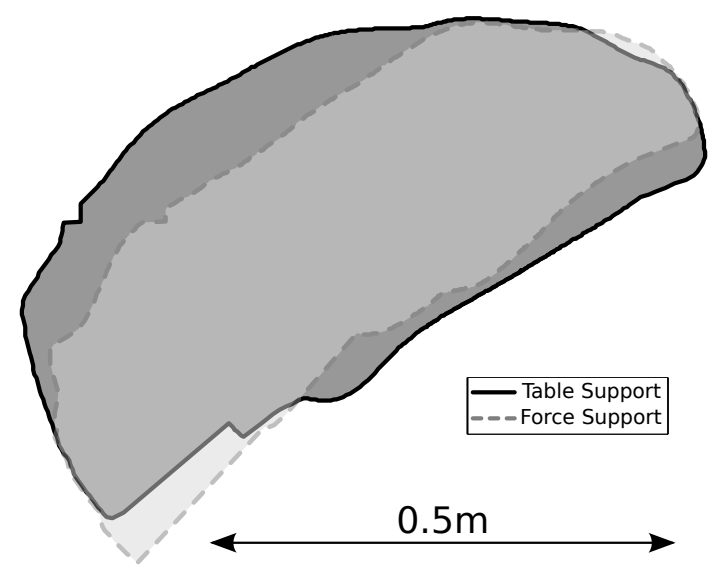

Fig. 1. Difference between table and full weight supports in a single case example. With the table support condition, a stroke patient has a larger range-of-motion (solid black line \& dark gray area), whereas with full weight support the reachable area is a lot smaller (stripped gray line \& light gray area). Data provided by Mike Ellis and Jacob McPherson.

vantages and disadvantages. In this short report, we wish to highlight these differences and detail the functional consequences.

\section{TABle Support versus Full Weight Support}

Weight-support systems function by either restricting vertical displacement or by supporting the arm with constant vertical forces. Planar devices like the MIT-Manus [10] support the weight of the arm by restricting all vertical displacement, which feels like forced sliding of the arm on a smooth horizontal surface. Although this fully supports an arm at rest, the amount of compensation is always equal to the normal force between the arm and the surface and cannot be scaled or externally controlled. Even when such planar mechanisms allow some upward displacements, patients need to fully support their arm themselves before any lift-off is achieved. In none of the aforementioned training studies [1], [2], [3], [4], [5] was a planar devices used; for all the slow reduction of weight support was an integral part of the therapy. One of these studies explicitly finds the progressive shoulder abduction loading to be a crucial element of arm rehabilitation in chronic stroke [5].

Secondly, patients can actively press their arm downwards in planar devices, potentially helping their achievements but hindering relearning of normal movement control. For example, using a table-like support artificially increases the range of motion of stroke patients over a fully force-supported arm [8] (see Fig. 1). This rewards patients for the erroneous motor 


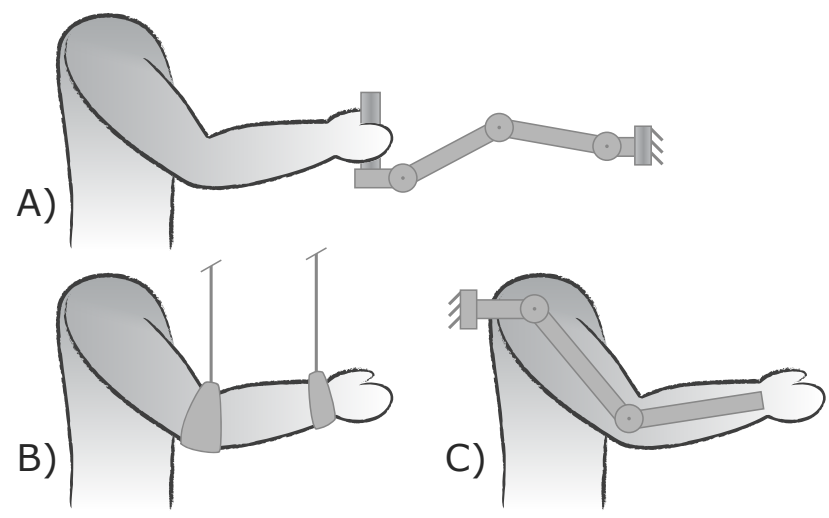

Fig. 2. Device types suitable to provide weight support. A) 3D endpoint manipulators. B) Cable suspension systems. C) Exoskeletons.

pattern of pushing down while wanting to achieve horizontal movements.

For these two reasons, planar endpoint manipulators seems less suitable for restoration of functional movements in stroke rehabilitation therapy. Full 3D endpoint manipulators [11], [12], [8], however, are fully capable of offering weight support through scalable forces.

\section{EXOSKELETONS VERSUS ENDPOINT MANIPULATORS}

For devices needing close control over the endpoint, exoskeletons or endpoint-manipulators are preferred over cable suspensions (see Fig. 2). Comparing exoskeletons and endpoint manipulators as an application mechanisms for transferring the supporting forces to the arm can be done on the variability of gravity compensation force in the workspace, the achievable range of motion, the movement impedance (mostly inertia and friction), the position and angle measurements, the ease of use, and finally, the ease of construction (including price and maintenance). For generic designs of these two types of rehabilitation devices, the following conclusions can be drawn.

Overall, exoskeletons do not score high marks as a dedicated weight support system. The main problem for the exoskeleton is the need of mechanics for all joint rotations, and the alignment of the exoskeleton joints to the anatomical ones [13], [14], [15]. Misalignment of axes and the additional two translational DOFs in the human shoulder require additional mechanics, making the exoskeleton more complex to construct and use. As an application mechanism, it has a relatively high impedance due to inertia and a limited range of motion. Control of supporting forces depends on the joint orientation. Exoskeletons not directly linked to the limb movements can use better force generating mechanisms with a larger range of movement [1].

Endpoint manipulators are easier to construct than exoskeletons and have a lower movement impedance. Having a mechanical system fully independent of the arm makes a constant force and a large range of motion easier to realize, although the device needs more workspace to operate in. Accurate position and angle measurements require recalculations from manipulator to arm coordinates and assumptions on the positions of trunk and/or shoulder.

Combination of application mechanisms have been used before. Many partial exoskeleton consist of endpoint mechanisms connection to a forearm cuff [16], often with reduced arm DOFs [8]. Some reduces the gravitational pull on their haptic endpoint device by supporting most of the weight of the arm by cable suspensions [12].

\section{Active Versus Passive Devices}

Another aspect to consider when providing weight support is wether to use active or passive actuation. All three devices types can be fitted with passive mechanisms like counter weights, springs, or spring mechanisms [17], [1], [9], although many rehabilitation robots also have active actuators like electro motors (partly) providing weight support [11], [12], [8], [15]. Advantages of passive weight support are the inherent safety of the device-patient interaction and no need for expensive electronics. Advantage of active support are the availability of fast, computerized control over the amount of weight support and the availability of negative support. With the latter, the training exercises can be made heavier beyond the limb weight, thereby simulating the weight of manipulated objects [8], [4], [5].

\section{CONCLUSions}

To facilitate upper-extremity movements, most rehabilitation devices support the arm against gravity. With weight support, patients may do more and more meaningful exercises earlier in the rehabilitation process.

There is a fundamental difference between weight support by limiting vertical displacement or applying constant supportive forces which counteract the gravitational pull on the arm. Using constant supportive forces is the most natural way to facilitate arm movements as it allows full freedom of movement and the amount of weight support is scalable to the patients needs.

To apply the supporting forces to the arm, endpoint mechanisms and exoskeletons are more complex to build and use then cable suspensions, but offer more control over the movements. This control is useful when combined weight support with other movement manipulation.

Finally, using passive weight support has the advantage of inherent safety, but actively-actuated weight support results in greater control of the support forces and the possibility to extend training environments to beyond limb-weight levels.

\section{REFERENCES}

[1] R. Sanchez, J. Liu, S. Rao, P. Shah, R. Smith, T. Rahman, S. Cramer, J. Bobrow, and D. Reinkensmeyer, "Automating arm movement training following severe stroke: functional exercises with quantitative feedback in a gravity-reduced environment." IEEE Trans Neural Syst Rehabil Eng, vol. 14, no. 3, pp. 378-389, 2006.

[2] M. Ellis, T. Sukal, T. DeMott, and J. Dewald, "ACT-3D exercise targets gravity-induced discoordination and improves reaching work area in individuals with stroke." in Proc 10th ICORR'07, B. Driessen, J. Herder, and G. Gelderblom, Eds., Noordwijk, the Netherlands, Jun 13-15 2007. 
[3] S. J. Housman, K. M. Scott, and D. J. Reinkensmeyer, "A randomized controlled trial of gravity-supported, computerenhanced arm exercise for individuals with severe hemiparesis." Neurorehabil Neural Repair, Feb 2009. [Online]. Available: http://dx.doi.org/10.1177/1545968308331148

[4] M. Ellis, T. Sukal, and J. Dewald, "Impairment-based 3d robotic intervention improves upper extremity work area in chronic stroke: Targeting abnormal joint torque coupling with progressive shoulder abduction loading." IEEE Trans Rob Autom, vol. in press, 2009.

[5] — , "Progressive shoulder abduction loading is a crucial element of arm rehabilitation in chronic stroke." Neurorehabil Neural Repair, vol. in press, 2009.

[6] G. Prange, L. Kallenberg, M. Jannink, A. Stienen, H. van der Kooij, M. IJzerman, and H. Hermens, "Influence of gravity compensation on muscle activity during reach and retrieval in healthy elderly." $J$ Electromyogr Kinesiol, 2007, epub.

[7] G. B. Prange, M. J. A. Jannink, A. H. A. Stienen, H. van der Kooij, M. J. Ijzerman, and H. J. Hermens, "Influence of gravity compensation on muscle activation patterns during different temporal phases of arm movements of stroke patients." Neurorehabil Neural Repair, Feb 2009. [Online]. Available: http://dx.doi.org/10.1177/1545968308328720

[8] T. Sukal, M. Ellis, and J. Dewald, "Shoulder abduction-induced reductions in reaching work area following hemiparetic stroke: neuroscientific implications." Exp Brain Res, vol. 183, no. 2, pp. 215-223, 2007.

[9] A. Stienen, E. Hekman, F. van der Helm, G. Prange, M. Jannink, A. Aalsma, and H. van der Kooij, "Freebal: dedicated gravity compensation for the upper extremities." in Proc 10th ICORR'07, B. Driessen, J. Herder, and G. Gelderblom, Eds., Noordwijk, the Netherlands, Jun 13-15 2007.

[10] N. Hogan, H. Krebs, J. Charnnarong, P. Srikrishna, and A. Sharon, "MIT-MANUS: a workstation for manual therapy and training. i," in Proc 2th ROMAN'92, Tokyo, Japan, Sep 1-3 1992, pp. 161-65.

[11] C. Burgar, P. Lum, P. Shor, and H. van der Loos, "Development of robots for rehabilitation therapy: The Palo Alto VA/Stanford experience." J Rehabil Res Dev, vol. 37, no. 6, pp. 663-673, 2000.

[12] R. Loureiro, F. Amirabdollahian, M. Topping, B. Driessen, and W. Harwin, "Upper limb robot mediated stroke therapy - Gentle/s approach.” Auton Robot, vol. 15, no. 1, pp. 35-51, 2003.

[13] A. Schiele and F. van der Helm, "Kinematic design to improve ergonomics in human machine interaction." IEEE Trans Neural Syst Rehabil Eng, vol. 14, no. 4, pp. 456-469, 2006.

[14] A. Stienen, E. Hekman, F. van der Helm, G. Prange, M. Jannink, A. Aalsma, and $H$. van der Kooij, "Dampace: dynamic force-coordination trainer for the upper extremities." in Proc 10th ICORR'07, B. Driessen, J. Herder, and G. Gelderblom, Eds., Noordwijk, the Netherlands, Jun 13-15 2007.

[15] T. Nef and R. Riener, "Shoulder actuation mechanisms for arm rehabilitation exoskeletons," in Proc Biorob'08, J. Desai, D. Meldrum, and O. Khatib, Eds., Scottsdale, Arizona, USA, Oct 19-22, 20082008.

[16] K. Nagai, I. Nakanishi, and T. Kishida, "Design of robotic orthosis assisting human motion in production engineering and human care." in Proc 6th ICORR'99, H. van der Loos, Ed., Stanford, California, USA, Jul 1-2 1999, pp. 270-275.

[17] J. Herder, "Development of a statically balanced arm support: Armon," in Proc. 9th International Conference on Rehabilitation Robotics ICORR 2005, 2005, pp. 281-286.

[18] B. Driessen, J. Herder, and G. Gelderblom, Eds., Proc 10th ICORR'07, Noordwijk, the Netherlands, Jun 13-15 2007. 